\title{
Efficient Cooperative Control System for pH Sensitive Nanorobots in Drug Delivery
}

\author{
S Y Ahmed \\ Department of Scientific \\ Computing, Ain Shams \\ University \\ Cairo, Egypt
}

\author{
S E Amin \\ Department of Scientific \\ Computing, Ain Shams \\ University \\ Cairo, Egypt
}

\author{
T Alarif \\ Department of Computer \\ Science, Ain Shams University \\ Cairo, Egypt
}

\begin{abstract}
In this paper, a control algorithm is proposed for $\mathrm{pH}$ sensitive nanorobots to deliver drugs in the tumor area while navigating in the blood stream environment. The nanorobots are able to communicate with their neighbors using the Particle Swarm Optimization algorithm. Furthermore, the obstacle avoidance algorithm allows the nanorobots to avoid collision with the blood cells. Each nanorobot can measure the $\mathrm{pH}$ value at its current position using sensors. Through cooperation, the nanorobots can drive the swarm to the tumor, which is defined by a certain $\mathrm{pH}$ value (less than 7.4). When the nanorobots locate the tumor cells, they release the drug which will raise the $\mathrm{pH}$ value of the cell until it is destroyed. The graphical interface simulations have shown the effectiveness of the proposed algorithm.
\end{abstract}

\section{General Terms}

Robotics, Artificial Intelligence, Nanotechnology

\section{Keywords}

Biomedical Nanorobots, Nanotechnology, Particle Swarm Optimization, pH Therapy, Reynold Number, Tumor

\section{INTRODUCTION}

Nanotechnology in the medical field can be classified as either being used for detection or for mediation. The motivation of nanotechnology as a behavior tool is derived from the opportunity to construct drug delivery systems or to modify the molecular arrangement of the disease by directly acquiring it. Various early theoretical analyses have been done to study the utility of the nanorobots for this [1].

These nanorobots are called biomedical nanorobots, which have attracted a lot of attention from scientists as they can benefit humans in numerous ways, incorporating physical support, cognitive support, social interfaces, and companionship. Conversely, control techniques over biomedical nanorobots are as of now difficult because of the recognized characteristic and need of having immediate communications with humans using expected social behaviors and standards.

Contrasting with mechanical robots, these robots must work safely while in the conditions that are expected. The issues developed in the cooperative control of biomedical nanorobots causes a motivation to solve them. It is important to comprehend the human environment and behaviors, and develop effective control techniques for biomedical nanorobots which is the general objective all through this paper.

The problem is studied for utilizing a swarm of nanorobots to deliver drugs to the tumor, which is a group level behavior that the individuals need their local and their neighbor's information. In this study, the tumor environment is considered as a scalar value field in space.

This research also concentrated on the cooperative movement of the nanorobots during navigation within the human body which is carried out by applying the swarm intelligence algorithm called Particle Swarm Optimization (PSO) [2] and the obstacle avoidance algorithm which is proposed in [3].

An important problem in cancer chemotherapy is the dangerous harmful side effects of the drugs on healthy cells. Consistently, the side effects require dose decreasing or treatment delay. It is challenging to control the harmful side effects of cancer chemotherapy on healthy tissues and to increase drug effectiveness to cancer. Researchers have improved various strategies using nanorobots to input cancer drugs into the human body. Cavalcanti et al. suggested several nanorobot architectures for medical nanorobotics $[4,5,6,7]$. In [8] swarm intelligence and chemical signaling techniques for nanorobots have been applied to the problem of drug delivery. Inspired by the theory from [8], Chandrasekaran and Hougen developed control techniques for nanorobot swarm intelligence [9], where the motion planning strategy is used to achieve swarm intelligent behaviors to achieve particular tasks.

In this research, the target area identification cooperative control strategy is simulated for $\mathrm{pH}$ sensitive nanorobots to deliver drugs in the tumor area. At a pH above 7.4 cancer cells become inactive and at $\mathrm{pH} 8.5$ cancer cells will die [10]. In our approach, nanorobots have been used to deliver the medicine which will raise the $\mathrm{pH}$ of the cancer cells as a cancer treatment, contributable to the concept of high $\mathrm{pH}$ therapy. One of the most important benefits of the multinanorobot strategy in medicine is that nanorobots can deliver sufficient therapy amounts and assist in keeping the right drug concentration. Moreover, cancer cells are very immovable and generally require more than one type of drug for the therapy. The multi-robot strategy meets this requirement, which can deliver multi drugs to the target area.

The rest of this paper is organized as follows. In Section 2, the nanorobot architecture and simulated environment properties are presented. In Section 3, the $\mathrm{pH}$ selective therapy approach is introduced. In Section 4, the control strategy is discussed that will allow the communication between the nanorobots to locate and reach the tumor target area without colliding against any obstacles. In Section 5, the simulation results are showed for a swarm of nanorobots used for tumor detection using the cooperative control strategies. Finally, this paper is concluded in Section 6. 


\section{NANOROBOT ARCHITECTURE AND ENVIRONMENT PROPERTIES}

The main objective in cancer therapy is to find tumor cells early to prevent their growth; hence, detecting tumors (targets for nanorobots) is an essential mission to achieve [11]. Especially, nanorobotics' architectures are associated with the progresses in nanomaterials of nanotechnology; where the use of these materials within the human body is impending [6]. In this section, the biomedical nanorobot architecture and the virtual environment properties will be discussed.

\subsection{Nanorobot Architecture}

A biomedical nanorobot generally is composed of a set of functionality modules, including chemical sensor, actuator, communication, and power supply [12]. These modules make the nanorobot capable in a particular medical application. Each module is supposed to take into account the hardware size, with its applicability for process inside the human environment.

Chemical Sensor: Nanosensors are used to transmit information about nanoparticles in the environment by computing changes in volume, concentration, velocity, gravitational, electrical and magnetic forces, pressure, or temperature of the cells in a human body [13].

Actuator: The electromechanical features of carbon nanotubes (CNTs) provide a chance toward the development of actuators. The properties of CNTs have been approved theoretically and experimentally [14].

For biomedical nanorobots, it is essential that actuators could be programmed to distinctive behaviors, which allow nanorobots to maneuver inside the human body.

Communication: In this paper, it is discussed how to navigate nanorobots to find the tumor and subsequently release drugs to destroy cancer cells. The nanorobot can only sense its local information, such as its current position and the $\mathrm{pH}$ value, but they do not know where the target is. For that reason, the most critical problem is to find a safe route to reach the target by communication with the other nanorobots which is achieved by using the PSO algorithm.

Power Supply: Power provides the energy to run sensors, actuators, and communications systems. A variety of power supply methods can be grouped into two categories, either from a source within the body or outside of the body. Molecule motor protein [14] and molecule motor DNA [15] nanodevices have been considered to perform transportation, chemical transformation, and enzymatic catalysis.

\subsection{Blood Stream Environment Parameters}

The most important goal of the environment model is to attain a working micro-world; therefore it must correspond to a scalable segment. The swarm nanorobots work to find the target information in the blood stream environment. Furthermore, each nanorobot has an extremely short sensing range. As a result, the virtual environment could be designed considering a blood vessel section only. The proposed parameters and value are listed in Table 1 .
Table 1. Blood Stream Environment Parameters and Values

\begin{tabular}{|l|l|}
\hline Parameter & Value \\
\hline Red cell radius & $7 \mu \mathrm{m}$ \\
\hline White cell radius & $12 \mu \mathrm{m}$ \\
\hline Blood viscosity $(\eta)$ & $10^{-2} \mathrm{~g} / \mathrm{cm} . \mathrm{s}$ \\
\hline Blood velocity $(v)$ & $1000 \mu \mathrm{m} / \mathrm{s}$ \\
\hline Blood density $(\rho)$ & $1 \mathrm{~g} / \mathrm{cm}^{3}$ \\
\hline Nanorobot radius & $3 \mu \mathrm{m}$ \\
\hline Nanorobot sensing radius & $10 \mu \mathrm{m}$ \\
\hline
\end{tabular}

The proposed blood stream environment has viscous and dynamic properties [3]. Furthermore, the modeled environment is created by internal interactions among the elements (red blood cell motion, red blood cell collision, fluid motion, and tumor growth). The inertial force (f) and viscosity $(\eta)$ of the blood [3] for a nanorobot affects its new positions. As a result, the Reynolds number $\mathrm{R}$ (the ratio of inertial to viscous forces for a nanorobot moving in blood) which is measured by Equation (1) which is used to adjust the velocity of the nanorobot.

$R=\rho f / \eta \quad$ (Where $\rho$ is density)

A constant velocity for the blood cells and the nanorobots is considered.

\section{THE pH THERAPY APPROACH}

The cells can only live within a certain $\mathrm{pH}$ range. The human body's $\mathrm{pH}$ values are influenced when eating and drinking. The $\mathrm{pH}$ number is an exponent number of 10 (see Equation 2). Consequently, a small change in $\mathrm{pH}$ translates to a big change in the amount of oxygen. In other words, blood with a $\mathrm{pH}$ value of 7.45 contains $64.9 \%$ more oxygen than blood with a $\mathrm{pH}$ value of 7.30 [16]

$p H=\log _{10} 1 / H$

The $\mathrm{pH}$ scale ranges from 0 to 14 , with numbers lower than 7.0 corresponding to an acidic (low oxygen) condition and upper than 7.0 corresponding to an alkaline, or oxygenated, condition.

Despite the fact that tumor cells have an increased acid production, they maintain normal cells with respect to healthy cells. The major acid load is transferred outside the cells; as a result, the extracellular space becomes acidic. Decreasing in $\mathrm{pH}$ presents a selective tumor marker for diagnosis and therapeutic treatments.

In this paper, the high $\mathrm{pH}$ therapy was used as a cancer medication, in which when the cancer cells were reached by the nanorobots they raised the $\mathrm{pH}$ of the cell. The cells that died were absorbed and eliminated by the body [10].

In the $\mathrm{pH}$ therapy approach, the nanorobots become active at low $\mathrm{pH}$, as a result of $\mathrm{pH}$ dependent release, in which the nanorobots are stable at $\mathrm{pH}$ 7.0-7.4. As the $\mathrm{pH}$ decreases to acidic $\mathrm{pH}(<\mathrm{pH} 7.0)$, the nanorobots release the drug which will raise the $\mathrm{pH}$ to 8.5 and the tumor will be destroyed [10].

Techniques for measuring the $\mathrm{pH}$ have been consistently improved. Based on these techniques, modeling $\mathrm{pH}$ sensitive nanorobots to locate tumor cells becomes achievable. The control algorithms are discussed in the following sections. 


\section{CONTROL ALGORITHM FOR THE NANOROBOT IN DRUG DELIVERY}

In this research, the environment is considered as a scalar value field in space, and it is assumed that each nanorobot has a $\mathrm{pH}$ sensor which can measure the $\mathrm{pH}$ value at its current position. The problem is that for each nanorobot in a swarm of nanorobots $r_{1}, r_{2}, \ldots . r_{n}$, locating at position $x_{1}(t), x_{2}(t), \ldots$, $\mathrm{x}_{\mathrm{n}}(\mathrm{t})$, with $\mathrm{pH}$ values, $\mathrm{pH}\left(\mathrm{x}_{1}(\mathrm{t})\right), \mathrm{pH}\left(\mathrm{x}_{2}(\mathrm{t})\right), \ldots, \mathrm{pH}\left(\mathrm{x}_{\mathrm{n}}(\mathrm{t})\right)$, design a control algorithm to move to the tumor target area, that is, the area of $\mathrm{pH}<7.0$ then release the drug which will raise the value of the $\mathrm{pH}$.

In this section, the swarm intelligence technique called Particle Swarm Optimization (PSO) is adopted for a group of nanorobots to cooperatively deliver drugs in the tumor. The obstacle avoidance algorithm presented in [3] also applied to the nanorobots for avoiding collision with blood elements during motion.

PSO is a comparatively new swarm intelligence optimization method developed by a social psychologist and an electrical engineer in 1995 [17]. This method is linked to artificial life in that it was developed to simulate the flocking of birds and swarming theory. The PSO algorithm starts by initializing a specific number of particles across the workspace. Particles have their own velocities that generate them around the workspace [18]. Each particle has information about two things: the location of the best it has ever attained, known as pbest, and the location of the best particle in the swarm, known as gbest, the global best. Both of these values are based on the objective function value. In each iteration, the velocity for every particle is updated using information about the location of its pbest and the current gbest of the swarm during when particles are searching through the workspace. Using the updated velocity, the position of the particle is updated in the workspace.

When the nanorobot moves on a new location, and verifies the presence of an obstacle, then, the obstacle avoidance algorithm is started. According to the distance between the nanorobot and the obstacle and the angle between the center of the nanorobot and the center of the obstacle, the nanorobot steps in the opposite or perpendicular direction of the obstacle.

The control algorithm for drug delivery to the tumor will proceed as follows, if a nanorobot doesn't detect an obstacle, it moves according to the PSO algorithm. While a nanorobot moves randomly, it can find the target area which has $\mathrm{pH}$ less than 7.0, and then it shares this value with its neighbor (so it will move to the best location based on this value). Otherwise, if an obstacle is encountered, a nanorobot follows the obstacle avoidance algorithm. After the nanorobot avoids the obstacle, a nanorobot moves again according to the PSO algorithm towards the tumor area.

The algorithm introduced in Table 2, where the ith nanorobot measures the $\mathrm{pH}$ value $\mathrm{pH}_{\mathrm{i}}$, position $\mathrm{x}_{\mathrm{i}}$ and velocity $\mathrm{v}_{\mathrm{i}}$, and receives the $\mathrm{pH}$ value, position, and velocity of all the other nanorobots by communication using PSO. This communication continues until one or more nanorobots have a measurement of the $\mathrm{pH}$ value less than 7.0 which indicates that a nanorobot reached the tumor area.
Table 2. Control Algorithm for Nanorobots in Drug Delivery

\begin{tabular}{|c|c|}
\hline 1. & For each nanorobot repeat \\
\hline 2. & If an obstacle is encountered \\
\hline $\begin{array}{l}3 . \\
\text { algorithm }\end{array}$ & Implement the obstacle avoidance \\
\hline 4. & Else \\
\hline 5. & Calculate $\mathrm{pH}_{\mathrm{i}}, \mathrm{x}_{\mathrm{i}}, \mathrm{v}_{\mathrm{i}}$ \\
\hline 6. & Implement the PSO algorithm \\
\hline $\begin{array}{l}7 . \\
\text { nanorobot }\end{array}$ & $\begin{array}{l}\text { Adjust the location and velocity of the } \\
t \text { according to the returned values } \mathrm{v} \text { and } \mathrm{pH}\end{array}$ \\
\hline 8. & UNTIL $\left(\mathrm{pH}_{\mathrm{i}}<7.0\right)$ \\
\hline $\begin{array}{l}9 . \\
\text { where can }\end{array}$ & $\begin{array}{l}\text { Release the drug, which raises the } \mathrm{pH} \text { value to } 8.5 \text {, } \\
\text { acer cells will be destroyed }\end{array}$ \\
\hline
\end{tabular}

\section{SIMULATION RESULTS}

The simulation scenario proposed here is about nanorobot operation in reaching the tumor cells in blood vessels. Through cooperation, the nanorobots can drive the swarm to the tumor which has a low $\mathrm{pH}$ value. The swarm will be used to deliver the medicine which will raise the $\mathrm{pH}$ of the cancer cells until being destroyed. The viscous forces, red cells and white cells of the blood must also be considered, as it will influence the nanorobots motion. Each time obstacles are recognized, the nanorobots locate to the new obstacle free positions. After an obstacle is avoided, each nanorobot will change its position so that the new position information and measure of $\mathrm{pH}$ are transmitted to the other nanorobots.

The tumor $\mathrm{pH}$ environment is simulated. Simulations were performed in a 2-dimensional space with a graphical interface as shown in Fig 1. The positions of the nanorobots, blood cells and target area, and also the specification of tumor cells is defined by the user.

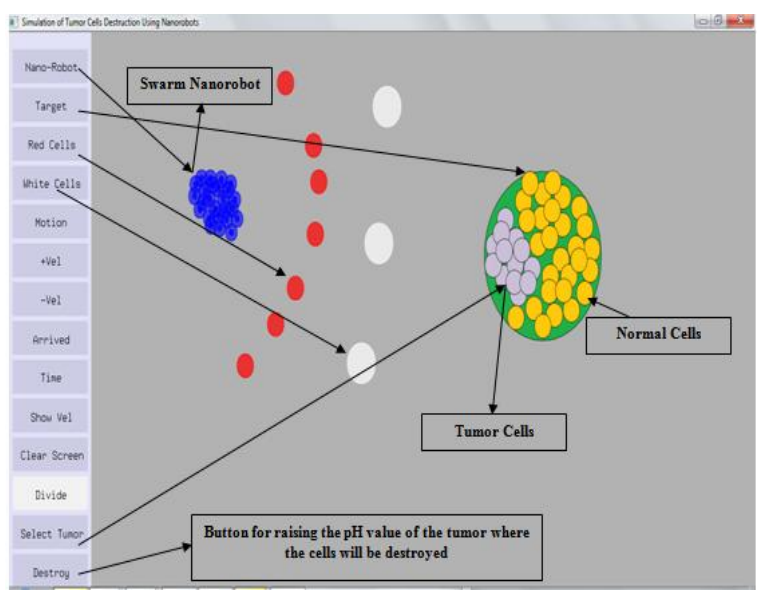

Fig 1: Representation of tumor $\mathrm{pH}$ environment in the drug delivery system 
Fig 2 introduces the scenario of a group of 25 nanorobots for drug delivery in tumor cells. After 40 seconds, the first nanorobot located the tumor position which has the lowest $\mathrm{pH}$ value, and transmitted it to its neighbors and so on. Thus, after 120 seconds, a group of 23 nanorobots reached the tumor as well, then the drug released and the $\mathrm{pH}$ increased. The Figure also shows the destruction of the tumor without harming the normal cells. So, the tumor cells are detected and destructed using $\mathrm{pH}$ sensitive nanorobots safely and in time. This indicates the excellent scalability of the proposed algorithm.

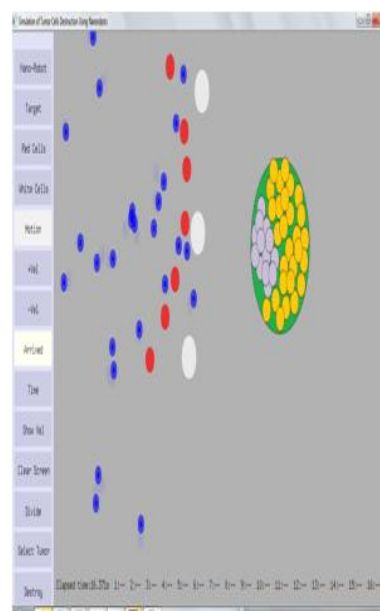

(a)

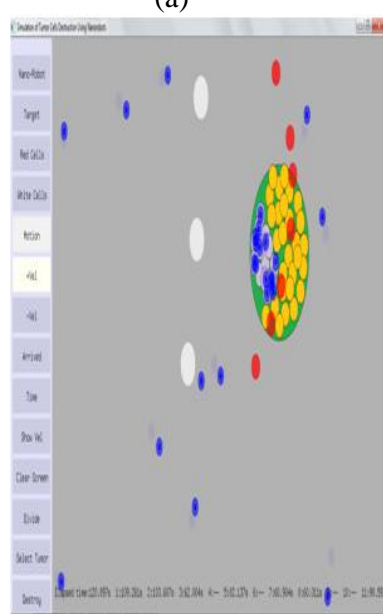

(c)

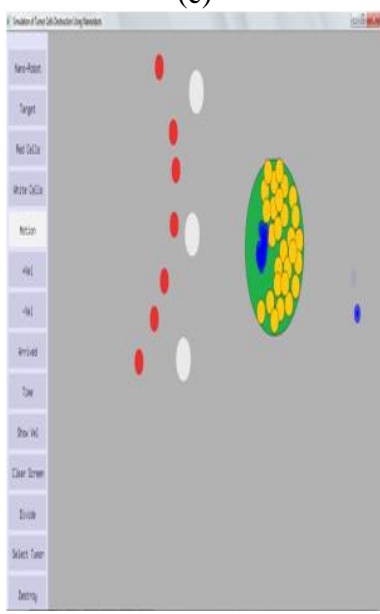

(e)

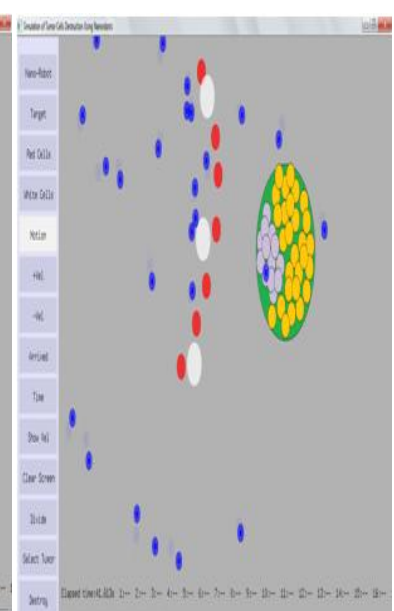

(b)

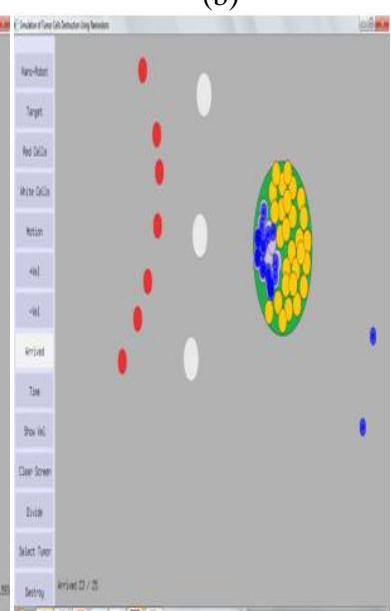

(d)

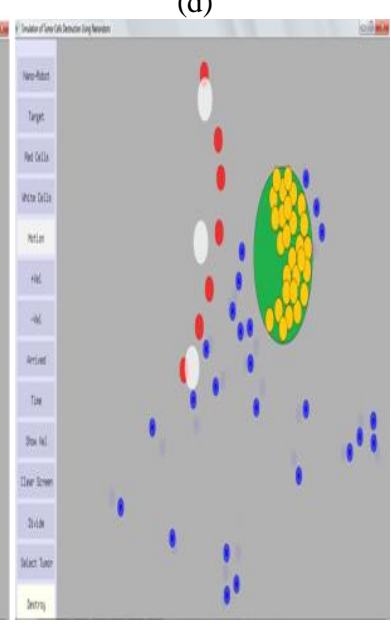

(f)
Fig 2: Simulation for the process of a group of $25 \mathrm{pH}$ sensitive nanorobots in the drug delivery system

\section{CONCLUSIONS AND FUTURE WORK}

In this paper, the biomedical nanorobot application was studied. The problem of using a swarm of nanorobots to deliver drugs to the tumor was considered. A control algorithm was implemented for $\mathrm{pH}$ sensitive nanorobots to trace the measured $\mathrm{pH}$ values, and reach the tumor cells with the lowest $\mathrm{pH}$ value. Cooperative control is performed by utilizing the swarm intelligence algorithm called Particle Swarm Optimization. The obstacle avoidance algorithm was also implemented to avoid collision with the blood elements in the nanorobot movements. The capability of the proposed algorithm was illustrated through simulating a scenario of the drug delivery by a swarm of nanorobots. Future work would include an enhancement on the design of the simulator by modeling the drug delivery system in $3 \mathrm{D}$ virtual reality of the human environment.

\section{REFERENCES}

[1] Adriano Cavalcanti, Robert A. Freitas, Jr. and Luiz C. Kretly, 2004, Nanorobotics Control Design: A Practical Approach Tutorial, ASME 2004 International Design Engineering Technical Conferences and Computers and Information in Engineering Conference.

[2] Bonabeau, E., Dorigo, M., Theraulaz, G.,1999. Swarm Intelligence: From Natural to Artificial Systems. Oxford University Press, New York.

[3] Khin Haymar Saw Hla, YoungSik Choi, Jong Sou Park, 2008. Obstacle Avoidance Algorithm for Collective Movement in Nanorobots, IJCSNS International Journal of Computer Science and Network Security, VOL.8 No.11.

[4] A. Cavalcanti and R. Freitas. Autonomous multi-robot sensor-based cooperation for nanomedicine. International Journal of Nonlinear Science and Numerical Simulation, August 2002.

[5] A. Cavalcanti and R. Freitas. Nanorobotics control design: A collective behavior approach for medicine. IEEE Transactions on Nanobioscience, June 2005.

[6] A. Cavalcanti, B. Shirinzadeh, R. Freitas, and T. Hogg. Nanorobot architecture for medical target identification. Nanotechnology, January 2008.

[7] A. Cavalcanti, B. Shirinzadeh, T. Fukuda, and S. Ikeda. Nanorobot for brainaneurysm. The International Journal of Robotics Research, April 2009.

[8] M. Lewis and G. Bekey. The behavioral selforganization of nanorobots using local rules. In Proceedings of IEEE International Conference on Intelligent Robots and Systems, 1992.

[9] S. Chandrasekaran and D. Hougen. Swarm intelligence for cooperation of bionano robots using quorum sensing. In Bio Micro and Nanosystems Conference, San Francisco, CA, June 2006

[10] K. Engin, D. Leeper, J. Cater, A. Thistlethwaite, L. Tupchong, and J. Mcfarlane. Extracellular ph distribution in human tumors. International Journal of Hyperthermia, 1995. 
[11] Dhariwal, A., Sukhatme, G.S., Requicha, A.A.G.: Bacterium-inspired Robots for Environmental Monitoring. In: Proceedings of the 2004 IEEE International Conference on Robotics and Automation, New Orleans, 2004.

[12] A. Ummat, G. Sharma, C. Mavroidis, and A. Dubey. Biomedical Engineering Handbook, chapter Bionanorobotics: state of the art and future challenges. CRC Press, 2005.

[13] R. Freitas. Nanomedicine, Volume I: Basic Capabilities. Landes Bioscience, Georgetown, TX, 1999.

[14] Y. Astier, H. Bayley, and S. Howorka. Protein components for nanodevices. Current Opinion in Chemical Biology, 2005.
[15] N. Seeman. From genes to machines: Dna nanomechanical devices. Trends in Biochemical Sciences, 2005.

[16] Y. Cui, Q. Wei, H. Park, and C. Lieber. Nanowire nanosensors for highly sensitive and selective detection of biological and chemical species. Science, 2001.

[17] Eberhart, R., Kennedy, J., 1995. A New Optimizer Using Particle Swarm Theory. In: Proceedings of the Sixth International Symposium on Micro machine Human Science, pp. 39-43. IEEE Press, New York.

[18] Siew Chin Neoh1, Norhashimah Morad2, Arjuna Marzuki1, Chee Peng Lim1, Zalina Abdul Aziz, 2009. A Multi-resolution GA-PSO Layered Encoding Cascade Optimization Model, Innovations in Swarm Intelligence. 